\title{
An Interesting Case of Fetal Cardiac Rhabdomyometa in Pregnancy
}

${ }^{1}$ GS Jyothi, ${ }^{2}$ Chaitra Shivananjaiah, ${ }^{3}$ NV Manjula, ${ }^{4}$ Asha Swarup

\begin{abstract}
Mrs S was detected to have fetal cardiac rhabdomyometa at 30 weeks of gestation, as the fetal echo showed multiple nonobstructive cardiac rhabdomyometa with normally connected heart and premature atrial contractions. Magnetic resonance imaging (MRI) showed no obvious cranial tubers. As the condition was isolated, patient was conservatively followed-up. Patient had an uneventful vaginal delivery. Post-delivery, fetal echocardiography showed multiple masses on the left and right side of the cardia on the tricuspid valve and was opined conservative management in view of high risk, had limited benefit for surgical intervention and as the condition would likely to improve over time. Baby was stable at discharge and was put on tablet propranolol $10 \mathrm{mg} 1 / 4$ thrice daily for selflimiting arrhythmias.
\end{abstract}

Keywords: Arrhythmias, Cardiac rhabdomyoma, Propranolol, TSC.

How to cite this article: Jyothi GS, Shivananjaiah C, Manjula NV, Swarup A. An Interesting Case of Fetal Cardiac Rhabdomyometa in Pregnancy. Int J Infertil Fetal Med 2015;6(2):92-95.

\section{Source of support: Nil}

Conflict of interest: None

Date of received: $22-05-15$

Date of acceptance: $30-06-15$

Date of publication: August 2015

\section{INTRODUCTION}

Fetal cardiac rhabdomyoma is a rare and most common primary pediatric tumor and constitutes more than $60 \%$ of antenatally detected cardiac tumors. ${ }^{1}$ For a fetus (unborn baby), the diagnosis accounts for $1 \%$ of all cardiac disorders detected by prenatal ultrasound. Incidence is 0.002 to $0.25 \%$ at autopsy, 0.02 to $0.08 \%$ in live-born infants and $0.12 \%$ in prenatal reviews, either sporadic or with certain genetic disorder. The natural history of cardiac rhabdomyometa is one of spontaneous regression. The fastest growth time for these tumors is between 2 nd and

\footnotetext{
${ }^{1,4}$ Professor, ${ }^{2}$ Postgraduate, ${ }^{3}$ Associate Professor

${ }^{1-4}$ Department of Obstetric and Gynecology, MS Ramaiah Medical College, Bengaluru, Karnataka, India

Corresponding Author: GS Jyothi, Professor, Department of Obstetric and Gynecology, MS Ramaiah Medical College Bengaluru, Karnataka, India, Phone: 09845257772, e-mail: drjyothigirish40@yahoo.co.in
}

the 3rd trimester. More than $85 \%$ of them are detected after 24 weeks of gestation. Growth slows as the pregnancy nears its end and stops when the baby is born. The biggest concern when a rhabdomyoma is seen on prenatal ultrasonography (USG) is its association with tuberous sclerosis which is seen in 40 to $90 \%$ of the babies.

Here, we report a case of fetal cardiac rhabdomyometa without tuberous sclerosis diagnosed antenatally and also discuss regarding the importance of diagnosing the condition in the prenatal period.

\section{CASE REPORT}

Mrs S, G2A1 23-year old, a well booked case, with second degree consanguinity was having regular uneventful antenatal care with us. Nuchat translucency-nasal bone and anomaly scans done were normal. She came with reduced fetal movements at 30 weeks of gestation. Nonstress test and biophysical profile done for fetal well being detected missed beats in the fetal cardia, and hence was advised fetal echocardiography. The fetal echocardiography done showed multiple nonobstructive cardiac rhabdomyometa with normally connected heart $1.5 \times 1.3 \mathrm{~cm}$ hyperechoic solid space occupying lesion arising from the free wall of the right ventricle noted just under the tricuspid valve leaflet. Similar, small $7 \mathrm{~mm}$ lesion seen in the free wall of the left ventricle. Every third atrial contraction was premature followed by a compensatory pause. 1:1 atrioventricular conduction sequence noted. Color Doppler study showed normal blood flow across the atrial and semilunar valves (Fig. 1). Due to a strong association between rhabdomyometa and tuberous sclerosis, magnetic resonance imaging (MRI) was done to rule out tubers in the brain, which showed no obvious cranial tubers or any significant abnormality, which was reassuring (Fig. 2).

There was no family history of tuberous sclerosis. Pediatric cardiologist opined that as the condition was isolated, the baby had a good prognosis and the lesions were likely to disappear over time and needed followup once in 2 weeks during pregnancy for heart rate and rhythm and also for tuberous sclerosis postnatally. Patient was monitored regularly with Doppler and nonstress test. Heart rate was maintained between 118 to 131 beats per minute. There was no supraventricular tachycardia. 
Patient delivered vaginally a healthy baby with good apgar scores at term and was shifted to neonatal intensive care unit (ICU) in view of atrial ectopics. As the saturation of the baby was falling, was put on antiarrhythmic drugs, after which the saturation picked-up to $80 \%$. Echocardiography done post-delivery showed multiple rhabdomyometa, moderate secondum atrial septal defect, right to left atrial ectopics seen with good biventricular function. Multiple soft tissue masses in right and left ventricle, predominately intramural, one large $22 \mathrm{~mm}$ close to the tricuspid valve partially obstructing the tricuspid valve inflow with no gradient, moderate tricuspid regurgitation and moderate to severe atrial regurgitation seen. Ultrasonogram abdomen and neurosonogram of the baby done was normal. Baby having rhabdomyoma of the tricuspid valve with increased right atrial pressure, with right to left flow and was advised conservative management. The baby was put on tablet propranolol $10 \mathrm{mg} 1 / 4$ tablet thrice daily for self-limiting arrhythmias (Fig. 3).

On day 7 of life, ECG was normal with sinus rhythm. Echocardiography showed ectopics, right sided mass on tricuspid valve, moderate tricuspid regurgitation, moderate secondum atrial septal defect with right to left flow and good biventricular function. Some amount of desaturation and tachyarrhythmia expected from cyanotic heart disease but surgical intervention had high risk and limited benefit. The location of the lesion on the cardiac valve was also crucial for surgical intervention and it was expected that the lesion would regress in early infancy.
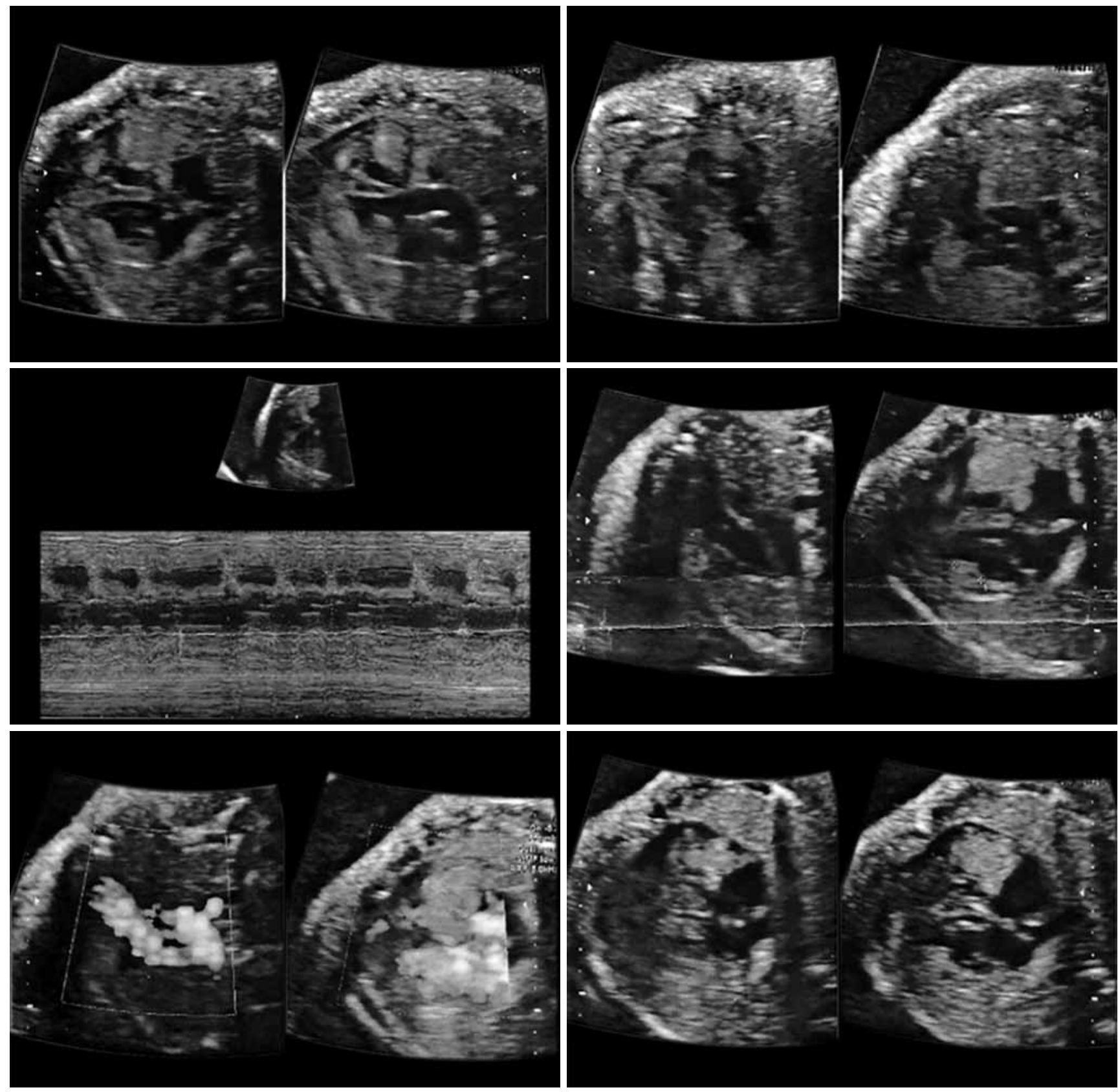

Fig. 1: Fetal echo showing multiple cardiac rhabdomyometa 


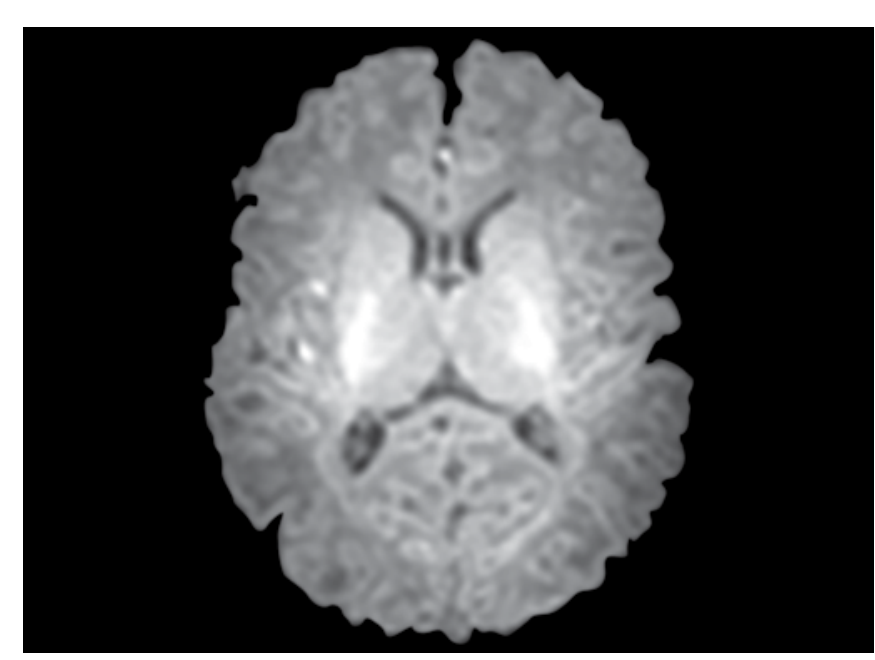

Fig. 2: Magnetic resonance imaging of the infant, with no features of tubes

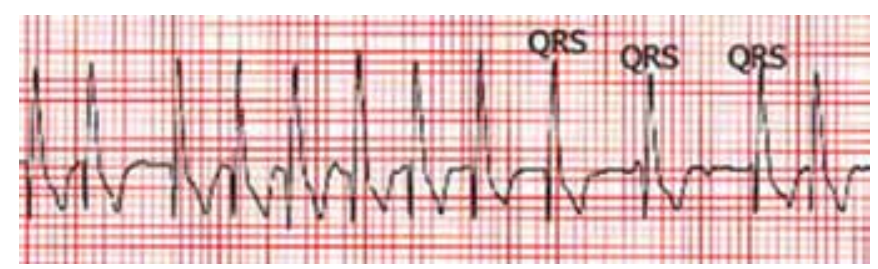

Fig. 3: Electrocardiogram showing atrial fibrillation (irregular RR interval)

The baby was discharged on tablet propranolol $10 \mathrm{mg} 1 / 4$ thrice daily and was monitored every week for 2 months and following that every month for 6 months. The baby was continued on tablet propranolol $2.5 \mathrm{mg}$ thrice daily which was changed to twice daily after 6 months of birth. It had appropriate milestones and came for regular follow-up until 6 months and was stable maintaining a saturation of around 80 to $90 \%$. Later on baby was lost for follow-up. At 11 months of age, baby had an episode of convulsion and was admitted to neonatal ICU of our hospital and detected to have an infarct of the right anterior cerebrum. Baby died after 3 days of admission and was on ventilator when it expired.

\section{DISCUSSION}

Cardiac rhabdomyometa are the most common cardiac tumors diagnosed in utero. Fetal cardiac tumors constitute one to two per 10,000 births. Around $90 \%$ of these are benign, out of which 60 to $80 \%$ are rhabdomyomas while others being fibromas, teratomas, vascular tumors and very rarely myxomas. ${ }^{2}$ They represent the earliest detectable hamartoma in tuberous sclerosis where they have been diagnosed in fetal life as early as 22 weeks of gestation. As they may be the only manifestation of tuberous sclerosis at this stage, probability that other features of the disease will develop with time is important for genetic counseling and patient management. ${ }^{3}$ The prenatal diagnosis of cardiac rhabdomyometa most often occurs after referral for the finding of a cardiac tumor or fetal dysrhythmia on routine obstetrical ultrasound assessment without other obvious features of tuberous sclerosis at the time of diagnosis, as also seen in our case. Knowledge of the outcome of affected fetuses and the true incidence of tuberous sclerosis in fetal cardiac rhabdomyoma is critical for accurate prenatal counseling and planning of prenatal treatment. ${ }^{4}$ When fetal cardiac rhabdomyometa is diagnosed, careful evaluation of other fetal structures including the brain and renal parenchyma should be performed to search for signs of tuberous sclerosis. ${ }^{5}$ The commonest complication associated with rhabdomyoma of the heart is conduction defect and obstruction. ${ }^{6}$

Tuberous sclerosis is an autosomal dominant disorder affecting approximately one in 6,000 to 8,000 individuals worldwide. Sporadic mutation constitute $50 \%$. Genetic disorder associated is tuberous sclerosis. Tuberous sclerosis is a hamartoma syndrome due to mutation in TSC1 or TSC2 in which cardiac rhabdomyometa are seen in approximately $60 \%$ of patients. The disease is characterized by the development of benign hamartomas in a number of organ systems and often has a prominent neurologic component due to the occurrence of CNS hamartomas that lead to epilepsy and developmental disorders. ${ }^{2}$ Frequently cardiac, renal, and lung lesions are asymptomatic and discovered at necropsy. There are also skin manifestations of the disease in the form of shagreen patches or adenoma sebaceum. In rare cases, it is associated with Tetralogy of Fallot (TOF), Ebstein abnormality, hypoplastic left heart syndrome, Down's syndrome and basal cell nevus syndrome. ${ }^{7}$ Detection of cardiac rhabdomyoma in the fetal period is important as they can lead to sudden death in some while they can regress spontaneously in the others. Cardiac rhabdomyometa are typically asymptomatic and are therefore, usually not operated upon unless they are obstructive, cause heart failure or are complicated with severe intractable arrhythmias. Also, they may be difficult to remove completely as they are often located deep in the myometrium, ${ }^{8}$ as was in our case. Serial observations have demonstrated that the majority of these lesions become less prominent over time, with some disappearing altogether as assessed by ultrasound. So, surgical resection is performed only when they cause life threatening complications. ${ }^{7}$

When symptoms are present, they are generally related to the size of the tumors and their location. So, asymptomatic tumors are simply monitored by echocardiography and holter recording, in addition to usual clinical examination. ${ }^{8}$ Two-dimensional echocardiography offers a simple noninvasive method, which shows cardiac tumors very well and is the most useful diagnostic test 
in pregnancy and early infancy. ${ }^{9}$ While most rhabdomyomas are multiple even a single lesion in infancy is very likely to be rhabdomyoma and if other features of tuberous sclerosis are found, the diagnosis is beyond reasonable doubt. ${ }^{6}$

A meta-analysis conducted by Chao et al showed that multifocality of fetal cardia rhabdomyometa and a positive history of tuberous sclerosis are two strong predictors of neonatal outcome. Large obstructive lesions and those interfering with cardiac motility also have a poor prognosis. ${ }^{3,10}$ Fetuses with a tumor of $\geq 20 \mathrm{~mm}$ in diameter and persistence of arrhythmias in the postnatal period increases the risk of neonatal and infant death. ${ }^{9}$ No embolic events have been reported and there is no need for oral anticoagulation in the absence of a specific indication like atrial fibrillation. ${ }^{8}$ A novel therapy of treating a patient with clinically significant cardiac rhabdomyometa is by giving Everolimus a mammalian target of rapamycin (mTOR) inhibitor. ${ }^{11}$

As we saw in our case, the low saturation was due to the mass leading to the shunting of blood from right to left and ectopics were due to conduction defects. Since our case was not associated with tuberous sclerosis, the outcome was considered to be good. In our case, the baby was not brought for regular check up after 6 months of age and expired at 11th month of age following a convulsion with a cerebral infarct. This case is reported because of its rarity and also it being an isolated lesion as it is not associated with tuberous sclerosis. Here, the infarct could be due to an emboli which is again very rare probably due to the crucial location of the rhabdomyometa on the cardiac valve.

\section{REFERENCES}

1. Serikawa T, Takahashi Y, Kikuchi A, Takakuwa K, et al. A case of infantile cardiac rhabdomyoma complicated by tuberous sclerosis. Cardiology Research 2010 Dec;1(1):24-26.

2. Ganesh C, Sridhar A. A case of fetal cardiac rhabdomyoma. Int J Infer Fetal Med 2013;4(2):66-69.

3. Webb DW, Thoma RD, Osborne JP. Cardiac rhabdomyomas and their association with tuberous sclerosis. Archives of Disease in Childhood 1993;68(3):367-370.

4. Bader RS, Chitayat D, Kelly E, Ryan G, et al. Fetal rhabdomyoma: prenatal diagnosis, clinical outcome, and incidence of associated tuberous sclerosis complex. J Pediatrics 2003;143(5):620-624.

5. Kelekci S, Yazicioglu HF, Yilmaz B, Aygun M, Omeroglu RE. Cardiac rhabdomyoma with tuberous sclerosis: a case report. PMID: 16130856 (PubMed-indexed for Medline).

6. Crawford DC, Garrett C, Tynan M, Neville BG, Allan LD. Cardiac rhabdomyomata as a marker for the antenatal detection of tuberous sclerosis. J Med Gen 1983;20(4):303-312.

7. Meikie L, McMullen JR, Sherwood MC, Lader AS, et al. A mouse model of cardiac rhabdomyoma generated by loss of Tsc1 in ventricular myocytes. Human Molecular Genetics 2005;14(3):429-435.

8. Benyounes N, Fohlen M, Devys J-M, Delalande O, Moures JM, et al. Cardiac rhabdomyomas in tuberous sclerosis patients: a case report and review of the literature. 1875-2136/S. Elsevier Masson France. DOI: 10.1016/J.acvd:2012.01.009.

9. Smith HC, Watson GH, Patel RG, Super M. Cardiac rhabdoomyomata in tuberous sclerosis: their course and diagnostic value. Archives of Disease in Childhood 1989;64:196-200.

10. Karatas A, Karatas Z, Ozlu T. Fetal cardiac rhabdomyoma without tuberous sclerosis: a case report. Int J Med SCI Public Health 2013;2(4);1095-1097.

11. Tiberio D, Tranz DN, Philips JR. Regression of a cardiac rhabdomyoma in a patient receiving everolimus. Pediatrics (Official Journal of the American Academy of Pediatrics) 2011;127:e1335; originally published online April 4, 2011. p. 1335-1337. 